\title{
Weight a minute - iatrogenic paracetamol toxicity is preventable by utilisation of well-designed drug charts
}

\author{
Amad Khan, Kathryn Flavin, Jason Tsang \\ Barts Health NHS Trust
}

\begin{abstract}
latrogenic Paracetamol toxicity is a potentially life-threatening yet avoidable cause of acute liver failure. Unfortunately, several cases have recently been reported nationally $(1,2)$. The impetus behind our project was a recent case of iatrogenic Paracetamol induced hepatotoxicity within our trust, a London-based District General Hospital. According to the British National Formulary, for adults weighing 10-50kg the intravenous (IV) dose is $15 \mathrm{mg} / \mathrm{kg}$ every 4-6hours (max. $60 \mathrm{mg} / \mathrm{kg}$ daily), not the usual $1 \mathrm{gram}$ QDS oral dose which is applied irrespective of weight (3). We audited 100 adult patients in April 2013 and re-audited in July 2013. Both of the randomly selected samples consisted of an equal number of surgical and medical patients, with an equal gender ratio. Data of interest included whether patients were on IV Paracetamol, appropriately dosed; if and when patients had been weighed during admission; and whether the WHO pain ladder of analgesia was followed. Identified shortcomings included patient weight on admission not being recorded, and IV Paracetamol dose adjustment not being made in patients $<50 \mathrm{~kg}$. 3 months were spent raising awareness of the importance to record patient weights and to dose-adjust IV Paracetamol when indicated. Patients weighed on admission improved from $37 \%$ to $68 \%(p<0.0001)$ and those on the inappropriate dose of Paracetamol fell from 18 ( $25 \%$ of the patients on Paracetamol) to $5(5.75 \%$ of the patients on Paracetamol) $\mathrm{p}=0.0013$. There was a marked improvement in the number of patients with the weight written on their drug chart from $27 \%$ to $53 \%$ post-intervention. $(p=0.0003)$ In conclusion, every patient should be weighed on admission. In order to prevent potential hepatotoxicity, staff should document patient weights on the drug charts and be aware of the fact that patients who weigh $<50 \mathrm{~kg}$ should be on a $15 \mathrm{mg} / \mathrm{kg} /$ dose of IV Paracetamol, not 1 gram QDS.
\end{abstract}

\section{Problem}

latrogenic Paracetamol toxicity is a completely avoidable cause of acute liver failure. Given the ubiquity of parenteral Paracetamol prescribing, a mishap could potentially affect any in-patient. According to the British National Formulary, for adults weighing $10-50 \mathrm{~kg}$ the IV dose is $15 \mathrm{mg} / \mathrm{kg}$ every $4-6$ hours (max. $60 \mathrm{mg} / \mathrm{kg}$ daily), not the usual 1 gram QDS oral dose which is regardless of weight (3). Our audit's primary objective was to assess whether IV Paracetamol was appropriately dosed. Given that correct dosing is weight-dependent, our secondary objective assessed whether patients were weighed, and in a timely manner. The time taken for patients to be weighed was audited against local trust guidelines, which state that every patient should be weighed within 24 hours, and weekly thereafter. Finally, since Paracetamol is the most commonly prescribed analgesic, assessment of adherence to the WHO Pain Ladder was a further secondary objective (4).

\section{Background}

Paracetamol (acetaminophen) is a common and widely prescribed medication, used both for antipyretic and analgesic relief. Indeed, Paracetamol is the first key step in the WHO pain ladder. Due to its clinical pervasiveness, inadvertent IV Paracetamol overdosing could potentially affect any in-patient (5). For this reason, we undertook a Quality Improvement Project to determine whether patients were routinely being weighed, and if Paracetamol was appropriately dosed, as per relevant weight dependent adjustment.
There are increasing reports of iatrogenic Paracetamol poisoning related to use of intravenous administration $(1,2)$. Much of the literature however, focuses on dosing errors of Paracetamol amongst the paediatric population $(5,6)$. Only one paper sought to examine (albeit partially), factors leading to such mistakes (7). In particular, Cavell highlights drug errors in the paediatric prescribing of IV Paracetamol due to confusion of drug unit conversions between $\mathrm{mg}$ and $\mathrm{g} / \mathrm{ml}$. To the authors' knowledge, no research paper or QIP to date has focussed on iatrogenic Paracetamol toxicity in adults, and preventable areas that lead to such errors. Therefore, in this QIP we aimed to identify avoidable factors related to IV Paracetamol prescribing, and to make provisions to stop future errors.

\section{Baseline Measurement}

100 adult in-patients were randomly selected in April 2013. The sample comprised of equal numbers of surgical and medical patients, and an equal gender ratio. Data were collected using a questionnaire developed following consultation with doctors, nurses and pharmacists (see "Audit tool").

Initial findings showed $25 \%$ of patients were on the inappropriate dose of IV Paracetamol. Figure 1 depicts the break-up of patients on Paracetamol by route administered. Only $37 \%$ of patients were weighed on admission, while $27 \%$ of the patients did not have their weight recorded on the drug chart. The WHO pain ladder was not followed in $15.8 \%$ of patients.

See supplementary file: ds3080.doc - "Audit tool" 


\section{Design}

The impetus to instigate the project was a recent unfortunate case of iatrogenic hepatoxicity, secondary to Paracetamol, reported locally within our trust. We conducted Plan-Do-Study-Act (PDSA) cycles. This elucidated two interrelated problems with Paracetamol prescriptions within the trust. Firstly, patients were not always being weighed in a timely fashion, leading to incorrect dosing. This was despite local trust guidelines stipulating that all patients should be weighed within 24 hours of admission, and weekly thereafter. Secondly, there was a lack of knowledge amongst healthcare professionals that IV Paracetamol is dose adjusted for patients weighing $<50 \mathrm{~kg}$.

Inter-disciplinary collaboration was a key feature of the project. Involvement of the trust's most senior pharmacist enabled effective communication with the pharmacy department, and helped with the design of the questionnaire. Discussion with nursing staff and a senior clinician further ensured triangulation of perspectives on safe prescribing and administration of IV Paracetamol.

To secure the sustainability of safe IV Paracetamol prescribing, we put up permanent posters highlighting our recommendations in all the wards. Furthermore, we made Paracetamol prescribing one of the mandatory topics at induction for all doctors joining the trust.

\section{Strategy}

Our intervention was a Quality Improvement Project, based on the Plan-Do-Study-Act (PDSA) model. Our first PDSA cycle helped us design our questionnaire, and operated as our pilot study. Feedback from doctors, nurses and pharmacists helped to refine the questionnaire. Our second PDSA enabled us to identify two main issues surrounding Paracetamol prescribing - patients not being weighed on time (or at all) and a lack of knowledge regarding IV Paracetamol dose. Salient feedback included the need for patients' weights to be written on drug charts in order to prevent dosing errors based on visual estimates. In PDSA Cycle 3 we gathered feedback from all healthcare professionals involved in patient care, which informed our improvement strategy (detailed below).

Notably, we had inadvertently excluded ward clerks in our initial feedback responses. By including ward clerks in PDSA Cycle 4, we realised that change could not effectively be implemented without making clerical staff equal stakeholders in delivering patient care. Indeed, feedback from ward clerks resulted in administrators taking the lead in prompting patients to be weighed in a timely manner, and for patient weight to be documented on drug charts.

After PDSA Cycle 4 we had identified shortcomings and spent the following 3 months disseminating our findings and improvement strategy. Multiple forums were addressed using a wide variety of communication channels. For example, a trust-wide email was released to both senior and junior healthcare professionals. Posters detailing Paracetamol dosing were installed in all hospital wards. More formally, we published our findings in a Trust-wide Newsletter and a Medicine Safety Team Newsletter. The mechanism for ensuring the availability of patient weight on drug charts was achieved by empowering ward clerks to check for patient weight data. We also successfully introduced the topic of weightdependent prescription of IV Paracetamol to the induction for all new doctors joining the trust. This was achieved via cooperation with the pharmacy lead for medical education.

\section{Results}

The post-intervention data were measured using the same questionnaire (see "Audit tool") with Chi-square testing used to look for statistically significant differences. The percentage of patients weighed on admission improved from $37 \%$ to $68 \%(p<0.0001)$.

Similarly, the number of patients on the inappropriate dose of Paracetamol fell from 18 (25\% of the total of 72 patients on Paracetamol) to $5(5.75 \%$ of the total of 72 patients on Paracetamol) with a $\mathrm{p}=0.0013$ (Figure 2).

Improvement was also seen in the adherence of the WHO pain ladder for analgesic prescription, with an increase from 69 (84.2\%) to $91(94.8 \%)$ patients $(p=0.036)$.

Finally, there was a large increase in the number of patients with the weight written on their drug chart, improving from $27 \%$ to $53 \%$ post-intervention $(p=0.0003)$. The graphical representation of this data is in Figure 3.

Both our objective results and the subjective positive feedback from staff, suggest that our efforts were successful in addressing the initial problems of IV Paracetamol prescribing.

See supplementary file: ds3079.docx - "Graphical Representation of Salient Data"

\section{Lessons and Limitations}

We learnt that quality improvement requires a multi-disciplinary and fully collaborative approach. Also, simple cost-effective measures can make a large impact on patient care and safety. Our changes were successful in improving patients being weighed in a timely fashion, and for weights to be written on drug charts. Availability of these clinical parameters has reduced unnecessary risks posed to patients requiring weight-dependent IV Paracetamol.

Barriers and challenges faced during the project included a lack of equipment for weighing patients being identified by senior nursing staff. Equipment resources were subsequently addressed. Responsibility for documentation of weight on the drug charts was another initial barrier. We made provisions for this by emphasising the need for all healthcare professionals to undertake this shared responsibility. Finally, a lack of knowledge and a lack of continuity were further challenges. Weight-dependent prescription of IV Paracetamol has now become a part of the induction for all new doctors joining the trust, addressing both of these concerns. Future challenges remain in how best to continue the implementation of 


\section{BMJ Quality Improvement Reports}

the framework our team has created. Provisions for the study to be repeated next year will allow us to assess if our quality

improvement strategy holds longevity.

\section{Conclusion}

Weighing patients in a timely fashion, and recording their weight so that it is easily accessible to prescribers, nurses and pharmacists alike, means prevention of potentially serious mishaps with weightdependent drugs. Our findings show that significant changes in healthcare professionals' attitudes and behaviours can be achieved using simple cost-effective measures. These include ensuring that patient weight is clearly documented on drug charts. Pertinent information about IV prescribing of Paracetamol can be shared via established communication channels, such as emails, newsletters, posters and presentations. Our work is generalisable to all general hospital in-patient services.

\section{References}

1. Berling I, Anscombe M, Isbister GK. Intravenous paracetamol toxicity in a malnourished child. Clin Toxicol (Phila). 2012 Jan;50(1):74-6.

2. Beringer RM, Thompson JP, Parry S, Stoddart PA. Intravenous paracetamol overdose: two case reports and a change to national treatment guidelines. Arch Dis Child. 2011 Mar;96(3):307-8.

3. British National Formulary (BNF). Joint formulary committee. Pharmaceutical Press 2014

4. Organisation W. Analgesic Ladder. World Health Organization; 1986

5. BMJ Best Practice - http://bestpractice.bmj.com/bestpractice/monograph/337/basics/epidemiology.html Accessed 20/01/2014.

6. Dart RC, Rumack BH.Intravenous acetaminophen in the United States: iatrogenic dosing errors. Pediatrics. 2012 Feb;129(2):349-53.

7. Cavell GF. A safer presentation of intravenous paracetamol is needed. Eur $\mathrm{J}$ Hosp Pharm doi:10.1136/ejhpharm-2012-000135 Letter.

\section{Declaration of interests}

None

\section{Acknowledgements}

Daniela Webbe, Martin Hamilton-Farrell 